\title{
Nutrients drive transcriptional changes that maintain metabolic homeostasis but alter genome architecture in Microcystis
}

This article has been corrected since Advance Online Publication and a corrigendum is also printed in this issue

Morgan M Steffen ${ }^{1}$, Stephen P Dearth ${ }^{2}$, Brian D Dill ${ }^{3,5}$, Zhou Li ${ }^{3,4}$, Kristen M Larsen ${ }^{1}$, Shawn R Campagna ${ }^{2}$ and Steven W Wilhelm ${ }^{1}$

${ }^{1}$ Department of Microbiology, University of Tennessee, Knoxville, TN, USA; ${ }^{2}$ Department of Chemistry, University of Tennessee, Knoxville, TN, USA; ${ }^{3}$ Chemical Sciences Division, Oak Ridge National Laboratory, Oak Ridge, TN, USA and ${ }^{4}$ Graduate School of Genome Science and Technology, University of Tennessee, Knoxville, TN, USA

\begin{abstract}
The cyanobacterium Microcystis aeruginosa is a globally distributed bloom-forming organism that degrades freshwater systems around the world. Factors that drive its dispersion, diversification and success remain, however, poorly understood. To develop insight into cellular-level responses to nutrient drivers of eutrophication, RNA sequencing was coupled to a comprehensive metabolomics survey of $M$. aeruginosa sp. NIES 843 grown in various nutrient-reduced conditions. Transcriptomes were generated for cultures grown in nutrient-replete (with nitrate as the nitrogen (N) source), nitrogen-reduced (with nitrate, urea or ammonium acting as the $\mathrm{N}$ sources) and phosphate-reduced conditions. Extensive expression differences (up to 696 genes for urea-grown cells) relative to the control treatment were observed, demonstrating that the chemical variant of nitrogen available to cells affected transcriptional activity. Of particular note, a high number of transposase genes (up to 81) were significantly and reproducibly up-regulated relative to the control when grown on urea. Conversely, phosphorus $(P)$ reduction resulted in a significant cessation in transcription of transposase genes, indicating that variation in nutrient chemistry may influence transcription of transposases and may impact the highly mosaic genomic architecture of M. aeruginosa. Corresponding metabolomes showed comparably few differences between treatments, suggesting broad changes to gene transcription are required to maintain metabolic homeostasis under nutrient reduction. The combined observations provide novel and extensive insight into the complex cellular interactions that take place in this important bloom-forming organism during variable nutrient conditions and highlight a potential unknown molecular mechanism that may drive Microcystis blooms and evolution.
\end{abstract}

The ISME Journal (2014) 8, 2080-2092; doi:10.1038/ismej.2014.78; published online 23 May 2014 Subject Category: Integrated genomics and post-genomics approaches in microbial ecology Keywords: cyanobacteria; metabolomics; nitrogen; transcriptomics; transposase; urea

\section{Introduction}

Blooms of Microcystis aeruginosa occur in freshwater lakes, rivers and estuaries worldwide. Accumulation of biomass and cyanotoxins complicate both the ecological and socioeconomic health of lakes and surrounding communities (Dodds et al., 2008). For decades, phosphorus (P) loading has been cited as the primary cause of bloom events (Schindler, 1977). This paradigm has begun to

Correspondence: SW Wilhelm, Department of Microbiology, University of Tennessee, Knoxville, TN 37996, USA.

E-mail: wilhelm@utk.edu

${ }^{5}$ Current address: Medical Research Council Protein Phosphorylation Unit, University of Dundee, Dundee, UK.

Received 19 December 2013; revised 2 April 2014; accepted 8 April 2014; published online 23 May 2014 shift in recent years, however, to recognize a potential role for nitrogen $(\mathrm{N})$ concentrations and chemical forms in driving bloom formation and biological community structure (Dolman et al., 2012). Although this shift remains hotly debated (Downing et al., 2001; Scott and McCarthy, 2010; Paerl et al., 2011; Paterson et al., 2011), it is clear that Microcystis exhibits a differential response when exposed to various $\mathrm{N}$-containing chemical species (Donald et al., 2011) and concentrations (Vézie et al., 2002), as well as various $\mathrm{P}$ concentrations both in the environment (Wilhelm et al., 2003; Davis et al., 2010) and in culture (Shen and Song, 2007; Harke et al., 2012). Specifically, urea appears to stimulate rapid biomass accumulation of Microcystis when it enters fresh water as fertilizer runoff (Dolman et al., 2012). Despite decades 
of research, the cellular mechanisms driving these notably different responses to N-reduction and composition versus P-reduction remain undiscovered.

In this study, we used high-throughput RNAsequencing (RNA-seq) to comparatively measure global changes in transcriptional patterns for cultures of Microcystis aeruginosa NIES 843 grown under different $\mathrm{N}$ and $\mathrm{P}$ nutrient regimes. Corresponding metabolic pools were measured in parallel for each culture to establish a comprehensive blueprint of the molecular response of Microcystis to nutrient concentration and chemical composition. These observations help to explain the complex response of Microcystis and toxigenic cyanobacteria to changing patterns in freshwater biogeochemistry worldwide. Moreover, they highlight a previously unknown effect of nutrient chemistry and concentration on the genomic architecture and thus the evolutionary trajectory of this globally important cyanobacterium.

\section{Materials and methods}

\section{Culture conditions}

M. aeruginosa NIES 843 cultures were grown in CT medium (Ichimura, 1971) modified to produce five separate nutrient conditions (in triplicate) and to allow for growth across all conditions. Control conditions were standard CT medium that contained $1.67 \mathrm{~mm}$ nitrate $\left(\mathrm{KNO}_{3}\right.$ and $\left.\mathrm{Ca}\left(\mathrm{NO}_{3}\right)_{2}\right)$ and $164 \mu \mathrm{M}$ phosphate $\left(\mathrm{Na}_{2} \beta\right.$-glycerophosphate). Preliminary experiments examining growth constraints on cultures at reduced nutrient concentrations established the limits of nutrient reduction that still allowed for comparable growth rates between treatments (Supplementary Table 1). The P-reduced treatment contained control levels of nitrogen and $82 \mu \mathrm{M}$ phosphate $\left(\mathrm{Na}_{2} \quad \beta\right.$-glycerophosphate). The three N-reduced treatments contained $178.9 \mu \mathrm{M}$ of the nitrogen source and $164 \mu \mathrm{M}$ phosphate $\left(\mathrm{Na}_{2}\right.$ $\beta$-glycerophosphate). The three nitrogen variants in the nutrient-limited medium tested were nitrate (denoted N-reduced (nitrate) hereafter), ammonium $\left(\mathrm{NH}_{4} \mathrm{Cl}\right)$ and urea. To facilitate growth in the urea treatment, this medium was also supplemented with $100 \mathrm{~nm} \mathrm{NiSO}$, as the urease enzyme requires nickel $(\mathrm{Ni})$ as a cofactor and this metal is not in the original medium (Mackerras and Smith, 1986). Cultures were grown under 12:12 light/dark illumination of $110 \mu \mathrm{mol}$ photons $\mathrm{m}^{-2} \mathrm{~s}^{-1}$ at $26^{\circ} \mathrm{C}$. Cultures were passaged three times in their respective medium before being harvested during mid-exponential growth to ensure they were physiologically equilibrated to growth conditions. Because of nutrient constraints imposed by growth on reduced $\mathrm{N}$ and $\mathrm{P}$, the N-reduced (ammonia) and P-reduced treatments could not reach an exponential growth rate that matched the control treatment (Supplementary Table 1). All treatments except the N-reduced (ammonia) treatment were able to reach cell densities to match the control (Supplementary Table 1). Chlorophyll $a$ fluorescence, used as a proxy for cell biomass, was measured daily on a Turner Designs TD-700 fluorometer (Sunnyvale, CA, USA). To allow for extraction of sufficient nucleic acid material, triplicate cultures $(150 \mathrm{ml})$ were grown in $500 \mathrm{ml}$, $1 \% \mathrm{HCl}$-washed flasks. These samples were harvested at the estimated point of maximum growth rate (Supplementary Table 1). Cells were harvested by centrifugation for $15 \mathrm{~min}$ at 8500 r.p.m. Samples were stored at $-80^{\circ} \mathrm{C}$ before RNA extraction.

\section{RNA isolation, sequencing and analysis}

Total RNA was extracted from samples collected at the beginning of the light portion of the $12: 12 \mathrm{~h}$ light cycle with the MoBio PowerSoil Total RNA Isolation kit (MO BIO Laboratories, Carlsbad, CA, USA). Yields varied between treatments, with the $\mathrm{N}$-reduced (urea and ammonia) treatments yielding less total RNA from the control treatment; however, reduced yields did not affect the total amount of mRNA sequenced (Supplementary Table 1). Residual genomic DNA was removed using the Ambion Turbo DNA-free kit (Austin, TX, USA). Total RNA was kept at $-80^{\circ} \mathrm{C}$ before sequencing. Initial RNA quality assessment was performed using a NanoDrop 1000 spectrophotometer (Thermo Fisher Scientific, Waltham, MA, USA). Subsequent sample processing, including rRNA subtraction, library prep and sequencing, were performed by the HudsonAlpha Genomic Services Laboratory (Huntsville, AL, USA). RNA quality was confirmed by quantification on a Qubit Fluorometer (Life Technologies, Grand Island, NY, USA). The Ribo-Zero Magnetic Gold kit was employed for rRNA subtraction from all samples (Epicentre Biotechnologies, Madison, WI, USA). Library preparation was performed according to specifications provided by Illumina, and samples were sequenced on the Illumina MiSeq platform (San Diego, CA, USA).

\section{Bioinformatic analysis}

All transcriptome libraries were aligned to the M. aeruginosa sp. NIES 843 genome (Kaneko et al., 2007) using CLC Genomics Workbench v6.0 (CLC bio, Cambridge, MA, USA). Default recruitment parameters were used, allowing only two mismatches per alignment. Data were normalized by total number of reads per library. Expression values were calculated as reads per kilobase per million and normalized using the 'By Totals' option in CLC Genomics Workbench and using default settings (Mortazavi et al., 2008). After normalization, Baggerly et al. (2003) weighted t-type test was used for all comparisons. Statistical cutoffs were established at $P<0.05$ and $P$-values were corrected for false discovery rate. Because of current controversy surrounding use of reads per kilobase per million 
(Dillies et al., 2013), normalization methods were validated using a series of established housekeeping genes (Supplementary Figure 1). The genome plot was generated using BRIG (Alikhan et al., 2011). Sequences are available in the National Center for Biotechnology Information (NCBI) short read archive (PRJNA229846). Taxonomic identities for non-Microcystis reads were generated using Meta PhlAn with the very sensitive local option for read-marker similarity (Segata et al., 2012).

Metabolome and proteome processing and analysis For metabolite analysis, $25 \mathrm{ml}$ aliquots of each treat were collected on $0.45 \mu \mathrm{m}$ nylon filters (Whatman, Pittsburgh, PA, USA), extracted in 40:40:20 HPLC grade methanol, acetonitrile and water with $0.1 \%$ formic acid, and analyzed using an UPLC-Exactive Plus Orbitrap mass spectrometer (Thermo Fisher) (Lu et al., 2010). Metabolites identities were determined in silico via exact mass and retention time matching using MAVEN that also calculated area counts for each compound (Melamud et al., 2010; Clasquin et al., 2012). For proteomic measurements, samples were analyzed by 2-dimensional liquid chromatography-mass spectrometry on an LTQOrbitrap-Velos (Thermo Fisher) following protein extraction and digestion, as described previously (Gobler et al., 2011). Cell pellets of $10 \mathrm{mg}$ were lysed and proteins extracted in guanidine, yielding $\sim 1 \mathrm{mg}$ of protein per sample. Then, $\sim 100 \mu \mathrm{g}$ of protein was loaded onto strong cation exchange chromatography for processing. Resulting tandem mass spectrometry spectra (MS/MS) spectra were searched against an $M$. aeruginosa database. Modified normalized spectral abundance factor values (Zybailov et al., 2006; Abraham et al., 2011) for identified transposases were extracted and visualized as a heat map using Perseus (http://www.perseus-framework.org/).

\section{Chlorophyll a and microcystin estimates}

Microcystin concentrations were measured for each culture according to previously established methods (Hotto et al., 2008). Chlorophyll a (a proxy for Microcystis biomass) measurements were performed using the fluorescence method for normalization of toxin and metabolome data analysis on an AU-10 fluorometer (Tuner Designs, Sunnyvale, CA, USA) (Welschmeyer, 1994).

Alkaline phosphatase (APase) activity measurements APase (E.C. 3.1.3.1.) activity measurement was performed in biological triplicate across all treatments with the procedure adapted for cyanobacterial culture assays from Ivanikova et al. (2007). A TD-700 fluorometer was used to make measurements (Turner Designs). APase activity was normalized to chlorophyll $a$ measured as stated above.

\section{Results}

Growth characteristics and extraction yield

Growth rates of reduced nutrient treatments were lower than the control treatment (Supplementary Table 1). Biomass after 10 days of growth was also reduced $(83.1 \%$ and $22.3 \%$, respectively) for the $\mathrm{N}$-reduced (ammonium) and P-reduced treatments compared with the control (Supplementary Table 1). RNA yields from all reduced nutrient treatments were lower than the yields from control samples. Despite variable yields, $500 \mathrm{ng}$ of each sample was used to construct sequence libraries (Supplementary Table 1). Microcystin concentrations were below the detection limit for all samples.

\section{Summary of sequencing output}

On average, the sequence libraries contained a mean of $\sim 1.1$ million 50-bp reads, with $>99 \%$ of sequences free of ambiguous bases (Supplementary Table 1). An average of $65 \%$ of sequences stringently recruited to the $M$. aeruginosa NIES 843 genome. The majority of the remaining unrecruited reads (average $30.2 \%$ of unrecruited sequences) were annotated as various other Microcystis spp. (Supplementary Figure 2). These reads likely represent the fraction that could not be aligned to the template genome because of two base-pair mismatches from the published sequence and were therefore excluded from the M. aeruginosa NIES 843 expression analysis. Co-occurring bacteria within these nonaxenic cultures were also identified and classified: all reads not mapped to the NIES 843 genome by CLC Genomics were identified using MetaPhlAn. Most abundant phyla are shown in Supplementary Figure 2; the N-reduced (urea) treatment differed most widely from the other samples, both in most abundance of Proteobacteria and the number of unaligned reads (Supplementary Table 1).

Complete coverage of the Microcystis genome across all treatments is shown in Supplementary Figure 3. The greatest number of reads predictably aligned to portions of the genome containing the $5 S$ rRNA and ssrA (10Sa RNA) genes.

Global transcriptional response of M. aeruginosa NIES 843 to nutrient variability

Transcriptomes of $M$. aeruginosa NIES 843 grown under variable nutrient conditions exhibited significantly different mean global transcription profiles. When compared with the nutrient-rich control treatment, $\sim 11 \%$ of genes (696) were significantly upregulated under the N-reduced (urea) treatment (Figure 1a), whereas 1.5\% (Figure 1b), 0.3\% (Figure 1c) and $0.02 \%$ (Figure 1d) of genes were upregulated under the N-reduced (ammonium), N-reduced (nitrate) and phosphate-reduced conditions, respectively. Conversely, $4.1 \%$ of genes under N-reduced (urea) treatment (Figure 1a), 2.0\% of 

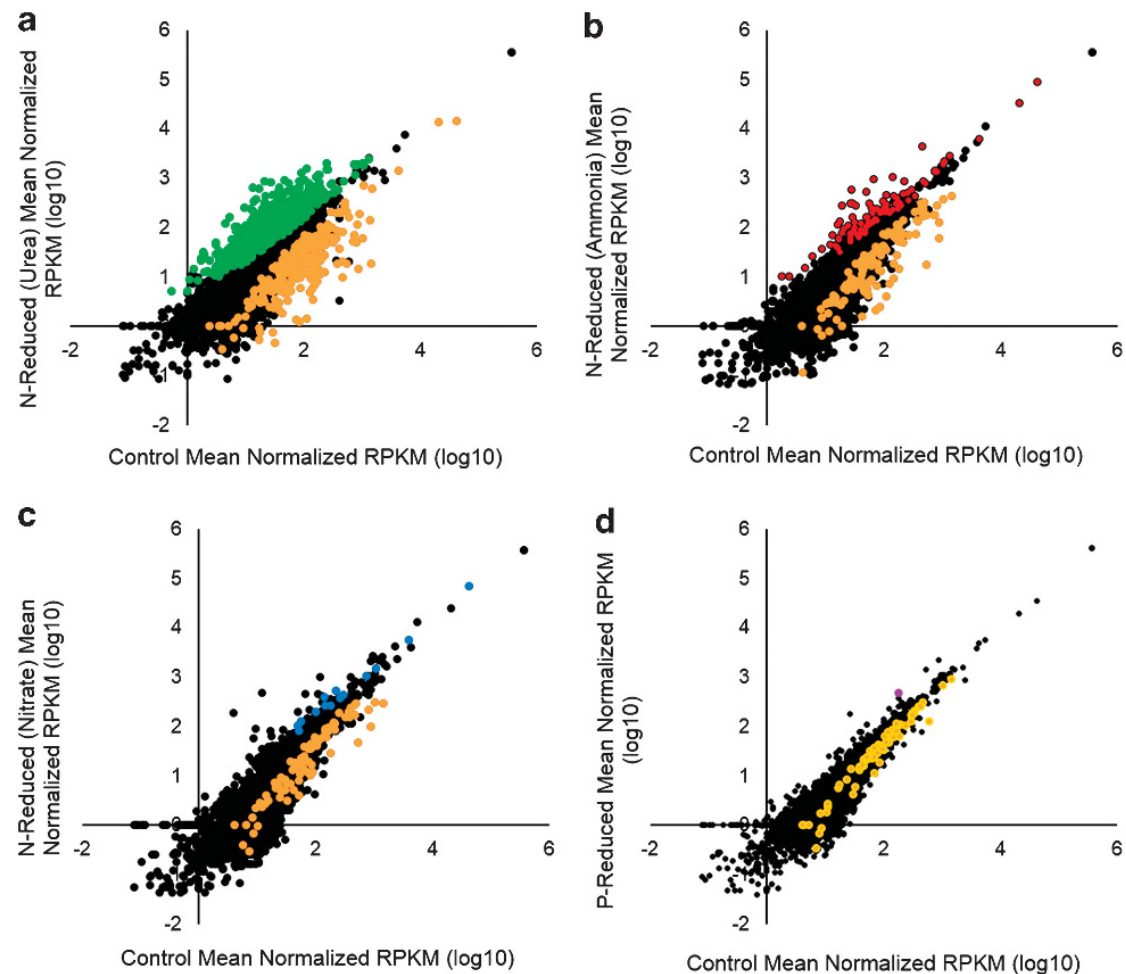

Control Mean Normalized RPKM $(\log 10)$

Figure 1 Comparison of global genomic expression between low nutrient treatments and the control treatment. Black points represent expression values of the 6364 genes of $M$. aeruginosa NIES 843. Orange points represent those genes that exhibit significantly decreased expression values in the low nutrient treatment transcriptome when compared with the control at $P<0.05$ (corrected for false discovery rate). Other colored points represent significant upregulation of expression values in the low nutrient treatment compared with the control at $P<0.05$ (corrected for false discovery rate). (a) Expression values of N-reduced (urea) vs control treatments; (b) expression values of N-reduced (ammonium) vs control treatments; (c) expression values of N-reduced (nitrate) vs control treatments; (d) expression values of P-reduced vs control treatments.

genes under N-reduced (ammonium) treatment (Figure 1b), 1.4\% of genes under N-reduced (nitrate) treatment (Figure 1c) and $1.1 \%$ of genes under low phosphorus (Figure 1d) were significantly downregulated compared with the control treatment. Drastic global transcription differences existed between the three $\mathrm{N}$-reduced treatments (Figure 2). For example, under the $\mathrm{N}$-reduced (urea) treatment, over 2000 genes were significantly upregulated in direct comparisons with either the N-reduced (ammonium) or N-reduced (nitrate) treatments (Figure 2).

Metabolic response of Microcystis to nutrient limitation We examined the expression of genes involved in $\mathrm{N}$ and $\mathrm{P}$ metabolism from the $M$. aeruginosa NIES 843 genome (NC_010296.1) to determine changes associated with alterations in nutrient availability and chemistry. Five genes involved in P metabolism and transport exhibited significant differential expression from the control under at least one low nutrient treatment. Expression of APase (MAE_16640) was upregulated under three out of four low nutrient treatments, although significant upregulation was observed only for the N-reduced (urea) treatment (Figure 3a). Three of four genes within the pathway (phosphate permease
MAE_09270, APase and phoU MAE_52660) experienced significantly increased expression compared with the control under the N-reduced (urea) treatment (Figure 3a). APase activity under these conditions was independently verified using an enzymatic assay, with only the N-reduced (urea) treatment exhibiting increased activity (Supplementary Table 1). Genes involved in urea metabolism largely exhibited reduced expression across all treatments when compared with the control treatment (Figure 3b). However, significant upregulation occurred for two of the three structural genes (ure $A-B$ ) of the urease enzyme complex (Figure $3 \mathrm{~b}$ ) in the N-reduced (urea) treatment. A gene involved in nitrate transport (MAE_14800) was significantly upregulated under the N-reduced (ammonium) treatment (Figure 3c). However, this transcriptional response was not emulated in the N-reduced (nitrate) treatment that only exhibited significant upregulation of nirA. Expression of the global nitrogen regulator gene, ntcA (MAE_01830), and the PII nitrogen regulator, $g \ln B$, were significantly different from the control under the N-reduced (urea) treatment alone (Figure 3c).

The water-soluble metabolome (that is, the pool of small molecules) within cells grown under these conditions was measured in parallel with each transcriptome. After comparison with the control 

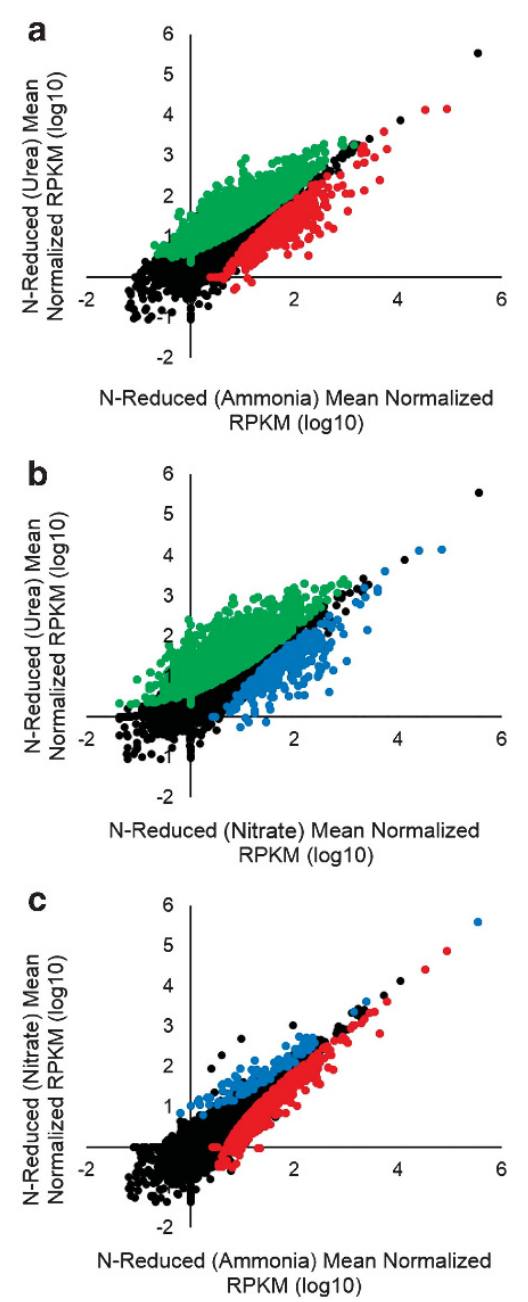

Figure 2 Comparison of global genomic expression between reduced nitrogen treatments. Black points represent expression values of the 6364 genes of $M$. aeruginosa NIES 843. Colored points represent significant up-regulation of expression in the specified low nitrogen treatment compared with the second at $P<0.05$. (a) Expression values of $\mathrm{N}$-reduced (urea) (green) vs $\mathrm{N}$-reduced (ammonium) (red) treatments; (b) expression values of $\mathrm{N}$-reduced (urea) (green) vs N-reduced (nitrate) (blue) treatments; (c) expression values of N-reduced (ammonium) (red) vs $\mathrm{N}$-reduced (nitrate) (blue) treatments.

cultures, relatively little variation between metabolomes among the N-reduced (urea) and other low $\mathrm{N}$ treatments were observed (Supplementary Figure 4). Outliers include $\mathrm{NADP}^{+}$, guanosine and NADPH (Figure 4 and Supplementary Figure 4). This pattern is echoed within pathways involved in $\mathrm{N}$ metabolism (Figure 4). In comparison with the control, metabolite pools largely respond in two ways: (1) N-reduced (ammonium and nitrate) and P-reduced treatments result in a significant increase in $\mathrm{N}$-acetylornithine, 2-oxoglutarate and malate; (2) the N-reduced (nitrate)-treated cells showed significant accumulation of certain metabolites (arginine, citrate, glutamate, glutamine and isocitrate) in comparison with the control treatment (Figure 4).
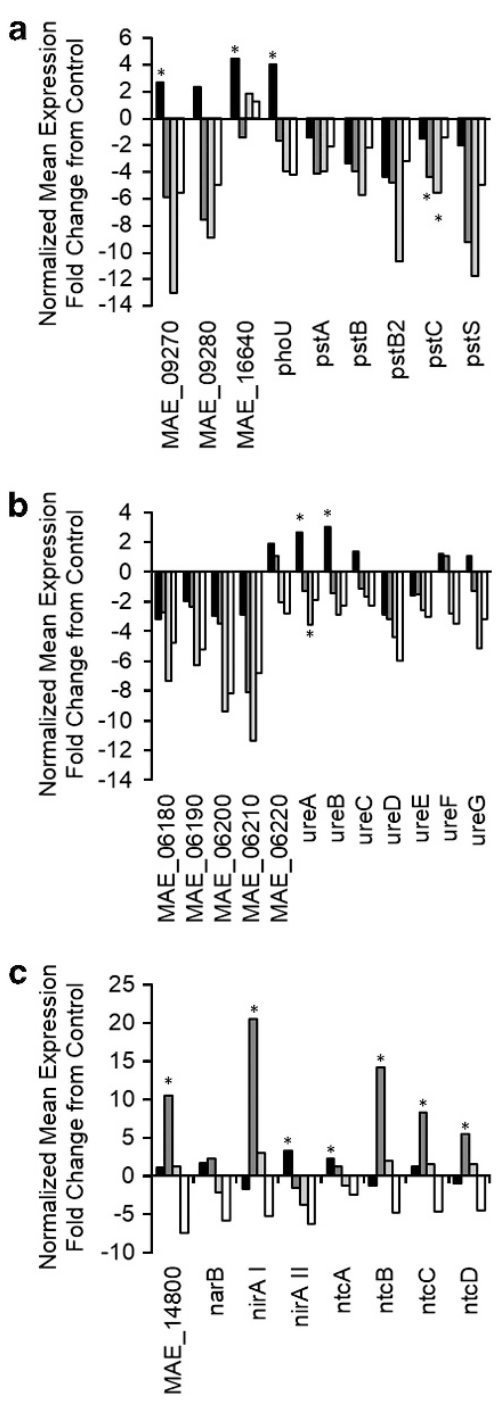

Figure 3 Fold change to expression values in comparison with control treatment of genes involved in $\mathrm{N}$ and $\mathrm{P}$ metabolism. ${ }^{*}$ Significant difference in expression value (RPKM) from control treatment at $P<0.05$. (a) Genes involved in phosphorus transport and metabolism; (b) genes involved in urea transport and metabolism; (c) genes involved in nitrate transport and metabolism. Black bars represent expression fold change of N-reduced (urea) treatment, dark gray bars represent N-reduced (ammonium) treatment, light gray bars represent $\mathrm{N}$-reduced (nitrate) treatment and white bars represent P-reduced treatment.

\section{Nutrients and toxin production by Microcystis}

The biosynthetic cassette encoding for the biosynthesis of microcystin toxin spans $55 \mathrm{~kb}$ of the genome and is composed of 10 genes. Transcription of all 10 genes was reduced in each low nutrient treatment compared with the control (Supplementary Figure 5). Functional assessment of toxin production was attempted by measurement of toxin concentration in each sample, but levels were below detection limits (data not shown). The genes involved in synthesis of cyanopeptolin $(m c n A-G)$ and aeruginosin $(a e r A-N)$ did not exhibit significant upregulation under any growth treatment. 


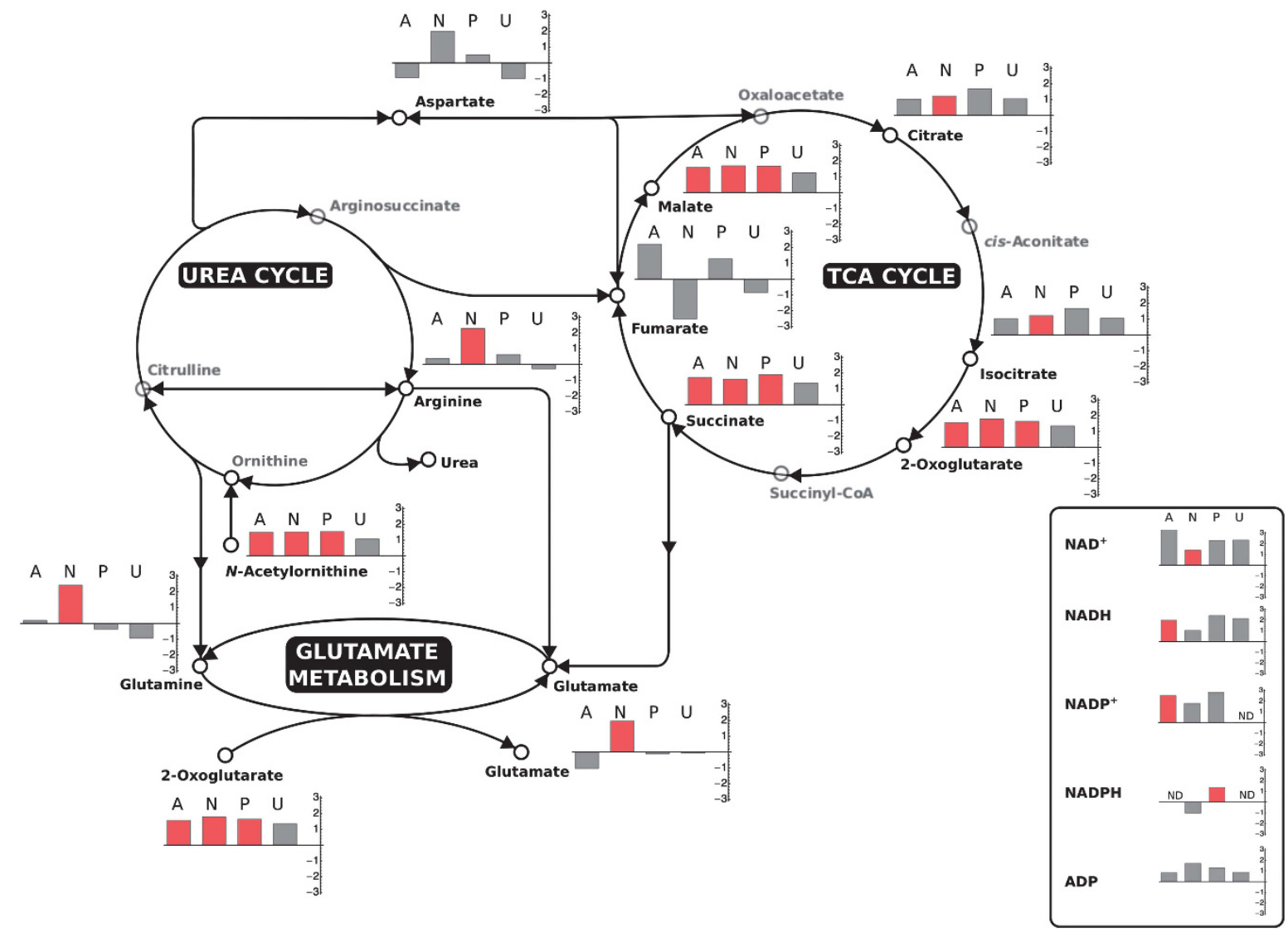

Figure 4 Metabolomic fold changes for treated samples compared with control samples displayed for relevant pathways. A, N-reduced (ammonium); N, N-reduced (nitrate); P, P-reduced; and U, N-reduced (urea). Bars represent $\log _{2}$ transformed fold intensity and directionality. Red indicates significant changes $(P<0.05)$.

Insights into novel genetic elements of Microcystis Approximately $9.6 \%$ of the M. aeruginosa NIES 843 genome comprises genes encoding transposases (Kaneko et al., 2007). Of these 610 genes, stark differences in transcription patterns were observed between treatments (Figure 5). Cells grown under $\mathrm{N}$-reduction with urea as the sole $\mathrm{N}$ source exhibited significantly increased and reproducible (mean coefficient of variation $=18.2 \%$ ) transcription of $12 \%$ of these transposase genes (Figure $5 \mathrm{a}$ ), whereas the N-reduced (ammonium) treatment showed 5.6\% of transposase genes significantly upregulated (Figure 5b) before false discovery rate analysis, which suggested the potential for 11 and 10 false positives, respectively. Exposure to P-reduced conditions had the opposite effect on transposase gene transcription; 58 genes were significantly reduced in transcription compared with control (Figure $5 \mathrm{~d}$ ). These observations were validated by proteomics that showed a similar pattern of increased transposase expression during N-reduction (nitrate) and a quieting of this expression during P-reduction (Supplementary Figure 6), although overlap of differential expression of specific genes from the control treatment was limited to two genes for the
N-reduced treatment and three for the P-reduced treatment.

Another anomaly within the M. aeruginosa NIES 843 genome is the existence of nine genes that have been previously annotated as putative nitrogen fixation elements (Kaneko et al., 2007): we consider these genes an anomaly as there is no evidence that this organism fixes nitrogen. Differential transcription of these genes was observed when comparing the mean transcriptomes of the five nutrient treatments (Table 1). Greatest up-regulation of transcription occurred when cells were grown on N-reduced (urea) treatment. When comparing the N-reduced (urea) culture transcriptomes with the other two $\mathrm{N}$-reduced treatments, upregulation of $\geq 20$ times occurred for transcription of multiple genes annotated as being involved in $\mathrm{N}$-fixation (Table 1 ). Transcription of a single gene (MAE_15560), encoding a NifU-like protein, was greater in N-reduced (ammonium) and N-reduced (nitrate) than in the N-reduced (urea) treatment. Compared with the P-reduced treatment, six of these genes experienced significantly increased transcription when grown in N-reduced (urea) conditions (Table 1). 


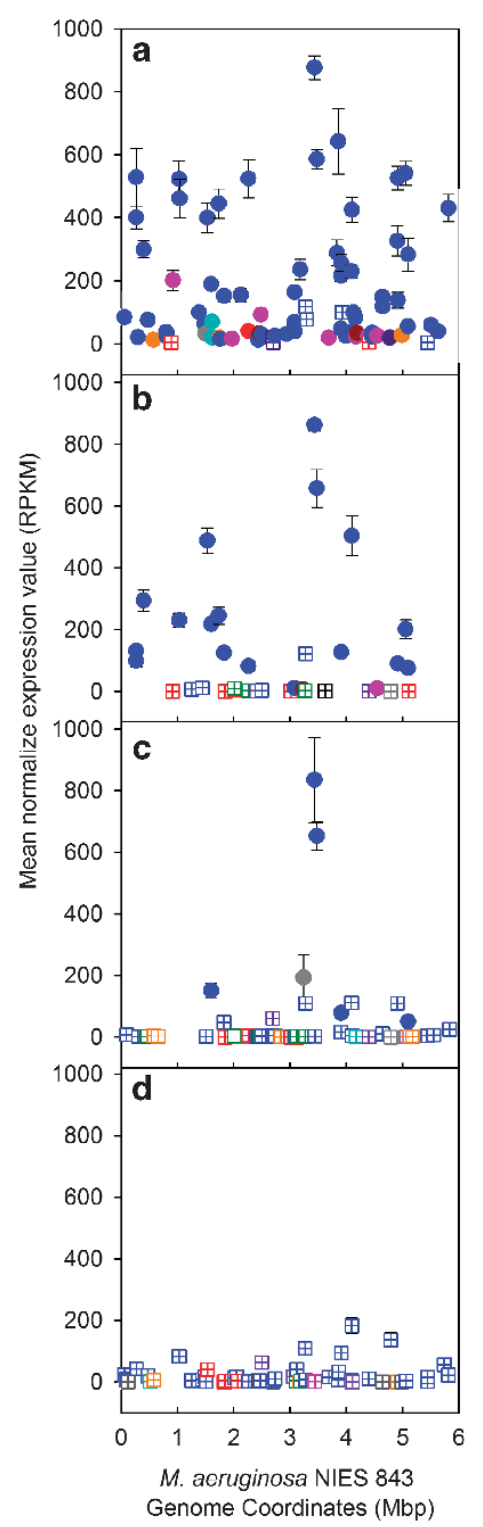

Figure 5 Mean normalized expression values (RPKM) of $M$. aeruginosa NIES 843 transposase genes with significantly $(P<0.05)$ different expression values from control treatment. Error bars represent s.e.m. of biological triplicates of (a) N-reduced (urea); (b) N-reduced (ammonium); (c) N-reduced (nitrate); and (d) P-reduced treatments. Circles indicate increased and squares decreased expression values from the control treatment. Colors represent transposase family: (IS200/IS605),

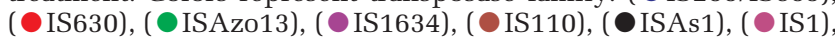
(○ISL3), (○ IS5), (○IS701) and (○ IS481).

\section{Discussion}

The insights gained from decades of research attempting to identify factors that drive cyanobacterial harmful algal blooms remain limited. Although factors such as temperature and nutrient availability have been implicated, their individual roles as drivers of freshwater blooms and phytoplankton speciation remain hotly debated (Scott and McCarthy, 2010; Paterson et al., 2011). We applied RNA-seq to replicate $M$. aeruginosa cultures to study the system-wide molecular response of this organism to alterations in nutrient concentration and chemistry. Our results demonstrate that widespread transcriptional changes are needed to maintain metabolic homeostasis within cultured Microcystis in response to nutrient variability. Moreover, significant and reproducible upregulation of transcription of transposases accompanied specific nutrient treatments, implying that anthropogenic nutrient loads may not only constrain biomass, but also could potentially shape the genomic architecture and ultimately the evolutionary trajectory of Microcystis populations. We discuss these results in the context of the ecology of one of the world's most common freshwater harmful algal species.

Insights into physiological responses of Microcystis to nutrient limitation

Genome-wide changes to gene transcription were observed when cultures were exposed to N-reduced as well as P-reduced conditions. Up to $\sim 20 \%$ of the annotated genes in nutrient-limited treatments were differentially transcribed compared with the control, suggesting expansive physiological responses to nutrient variability. Harke and Gobler (2013) demonstrated that additional strains of Microcystis also exhibit massive differential transcription patterns $(\sim 32 \%)$ in response to reduced $\mathrm{N}$ conditions. The most extensive changes in the current study were observed for cultures grown in N-reduced (urea) conditions (Figure 1a). Unexpectedly, the differences between the N-reduced (urea) treatment and the alternative N-reduced treatments were even more extreme (Figure 2). The changes in global transcription patterns suggest that the transcriptional response to $\mathrm{N}$ reduction by Microcystis is dependent on the chemical species of nitrogen available to the cell. In contrast with N-reduced treatments, relatively limited transcriptional change was observed under growth on $\mathrm{P}$ reduction relative to the controls. This limited response indicates that a more extreme reduction in $\mathrm{P}$ concentrations is necessary to upregulate physiological $\mathrm{P}$ stress in Microcystis cells. Capturing this response using advanced molecular tools such as RNA-seq represents a developing area of interest in HAB (Harmful Algal Bloom) research (Wurch et al., 2011; Harke and Gobler, 2013).

Genes involved in N metabolism exhibited anticipated responses to nutrient limitation. Specifically, genes involved in urea metabolism (ure $A-B$ ) were significantly upregulated during growth on urea (Figure $3 \mathrm{~b}$ ). This upregulation is supportive of the ability of Microcystis to use urea as its sole N source and indicates the use of the urease enzyme for the breakdown of the compound. This observation is salient to current agricultural trends, as the application of urea fertilizers is rapidly increasingly worldwide, resulting in elevated levels of urea in 
Table 1 Genes of the Microcystis aeruginosa NIES 843 genome annotated as having a function related to $\mathrm{N}_{2}$ fixation and the fold change in normalized expression values (RPKM) relative to the N-reduced (urea) treatment

\begin{tabular}{|c|c|c|c|c|c|}
\hline Gene & Function & Control & $\begin{array}{l}N \text {-reduced } \\
\text { (ammonium) }\end{array}$ & $\begin{array}{l}N \text {-reduced } \\
\text { (nitrate) }\end{array}$ & P-reduced \\
\hline MAE_14230 & Sigma factor potentially involved in expression of $\mathrm{N}_{2}$ fixation genes & 5.9 & 4.9 & 6.1 & 6.1 \\
\hline MAE 14860 & Putative transcription factor for heterocyst differentiation & - & 21.8 & 56.4 & 52.5 \\
\hline MAE_15560 & NifU-like protein & - & -1.2 & -1.4 & - \\
\hline MAE_16560 & Nitrogen regulation protein NifR3-like protein & - & 11.5 & 22.1 & 9.8 \\
\hline MAE_16580 & Nitrogen regulation protein NifR3-like protein & 2.7 & 16.5 & 13.0 & 33.6 \\
\hline MAE_18160 & Mo-dependent nitrogenase-like & - & 1.7 & - & - \\
\hline MAE 27840 & Heterocyst glycolipid synthase & 5.6 & 19.5 & 30.4 & 38.0 \\
\hline MAE_31880 & Mo-dependent nitrogenase-like & 2.2 & 2.7 & 4.5 & 9.6 \\
\hline MAE 38090 & NifY-like protein & - & 2.7 & 4.4 & 5.5 \\
\hline nifS & NifS cysteine desulfurase & - & - & 2.3 & - \\
\hline $\operatorname{sig} D$ & $\begin{array}{l}\text { Sigma factor potentially involved in expression of } \mathrm{N}_{2} \text { fixation genes } \\
\text { and light/dark adaptation }\end{array}$ & 2.8 & 4.3 & 8.4 & 8.3 \\
\hline
\end{tabular}

Abbreviations: N, nitrogen; P, phosphorus; RPKM, reads per kilobase per million.

agricultural runoff into aquatic systems (Glibert et al., 2006). Surprisingly, a significant upregulation of genes involved in phosphate transport and metabolism occurred under the N-reduced (urea) treatment and was not seen during $\mathrm{P}$ reduction (Figure 3a). The differences in APase transcription and activity under N-reduced (urea) limitation were particularly unexpected, as this gene is commonly used as an environmental marker for phosphorus limitation (Berman, 1970; DeBruyn et al., 2004). The lack of upregulation under the P-reduced treatment is potentially a result of experimental conditions designed to upregulate $\mathrm{P}$ stress not being limiting enough. In the case of Microcystis, deeper P-limiting conditions may be required for widespread upregulation of genes involved in phosphate metabolism, including APase (Healey and Hendzel, 1979; Harke and Gobler, 2013). Previous studies have found that APase activity is independent of phosphate concentrations when they are above $0.2 \mu \mathrm{M}$ in the environment (Nausch, 1998), much lower than those used in this study, and may in fact be linked to cellular N/C ratios in phytoplankton (Myklestad and Sakshaug, 1983; Neddermann and Nausch, 2004).

Because the N-reduced (ammonia) treatment was not able to reach cell densities comparable to the other treatments, transcriptional responses unique to this treatment may be reflective of a response to differences in cell densities, rather than nitrogen conditions. In addition, to determine whether supplementation of the N-reduced (urea) treatment with $\mathrm{NiSO}_{4}$ potentially confounded results, transcription patterns of genes involved in construction of enzymes requiring a $\mathrm{Ni}$ cofactor were examined. Only hoxH of the bidirectional hydrogenase (hoxEFHUY) and ureAB (ureA-G) showed significantly increased transcription in the N-reduced (urea) treatment compared with the control. Genes involved in the construction of the uptake hydrogenase (hypA-F), lactoylglutathionine (MAE_23250) and coenzyme F420 hydrogenase (MAE_40060) showed no difference between the urea and control treatments. Notably, the genes involved in $\mathrm{Ni}$ incorporation for urease (ureE) and hydrogenase (MAE_57880) showed no significant difference between the urea treatment and the control. Overall, these caveats highlight an important consideration often missed in single-variable experiments: metabolic pathways and genetic pathways do not function in isolation, but are tightly coupled. Along with the above considerations (that is, cell density effects on light-field, effects of growth using urea on $\mathrm{Ni}$ use and transport), there are innumerable other traits (for example, iron use and uptake for $\mathrm{NO}_{3}$ assimilation, zinc use and uptake associated with APase, the influence of nutrient chemistry on co-occurring/ contaminating microbes and so on) that can 'confound' observations in studies with microbial isolates, but are important considerations for such culture studies. Although it is likely that many of these effects are secondary to the more dramatic influences of shifts in nutrient availability, they provide a series of caveats that need to be remembered in the interpretation of our data.

The transcriptome and metabolome capture distinct levels of physiological response to environmental change, but do not always overlap. This is in part because of differences in turnover times for mRNA versus small metabolites (Spura et al., 2009; Steglich et al., 2010). Moreover, it is likely that many of the transcriptional responses are occurring in order to stabilize cellular metabolism because of environmental change. We therefore must exercise caution when comparing these measurements. In addition, metabolite biosynthesis and accumulation can become convoluted, as expression of multiple pathways can result in metabolic alterations that involve a single compound.

It is, however, possible to draw certain comparisons. For example, accumulation of lysine, an essential amino acid, occurred in cells grown in $\mathrm{N}$ reduction (urea) (Supplementary Figure 4). This is also reflected in the transcript data, with a gene involved in lysine biosynthesis ( $(a p B)$ significantly 
upregulated compared with the control treatment. When looking across the pathways highlighted in Figure 4, relatively few changes to metabolite pools can be observed between nutrient-limited treatments, especially in comparison with those occurring between the transcriptomes (Figures 1 and 2). These differences between the transcriptome and metabolome suggest that widespread transcriptional changes are necessary to maintain metabolic homeostasis when cells are exposed to nutrient limitation.

\section{Interactions with co-occurring heterotrophs}

Cultures used in this study were nonaxenic, and therefore interactions with the co-occurring heterotrophic bacterial community have the potential to indirectly shape gene transcription response to nutrient concentration. Of note, the drastic transcriptional response observed in the $\mathrm{N}$-reduced (urea) treatment coincided with the greatest number of unrecruited sequences to the NIES 843 genome (Supplementary Table 2). Heterotrophic transformation of organic $\mathrm{N}$ compounds, such as urea, to inorganic $\mathrm{N}$ has been shown to play an important role in the utilization of organic nitrogen by cyanobacteria in aquatic environments (Berman et al., 1999; Purvina et al., 2010). The active heterotrophic population in the cultures examined in this study may be fulfilling that role for Microcystis, as has been observed previously (Purvina et al., 2010). The contribution to urea metabolism by the heterotrophic population may explain why genes encoding urea transporters did not exhibit significantly increased transcription in the N-reduced (urea) treatment in Microcystis cells, as it may have been metabolized by contaminating heterotrophs before incorporation by Microcystis cells. More broadly, the transcriptionally active heterotrophic population in the N-reduced (urea) treatment may have had other indirect impacts on the global transcriptional response of Microcystis (for example, competition for $\mathrm{Ni}$ ) and could possibly explain at least a portion of the differences between the N-reduced (urea) and the alternative treatments.

\section{Genomic consequences of nutrient conditions}

The genomic architecture of Microcystis aeruginosa is considered to be among the most plastic of all cyanobacteria. Comprising $\sim 10 \%$ of the entire genome, the number of insertion sequence elements of $M$. aeruginosa outnumbers those found in the other bloom formers, including Nostoc punctiforme $(2.0 \%)$, Anabaena variabilis $(1.4 \%)$ and Cylindrospermopsis raciborskii $(0.9 \%)$ (Kaneko et al., 2007; Frangeul et al., 2008; Lin et al., 2011; Humbert et al., 2013). Indeed, the presence of repetitive sequences and associated transposase genes have likely made successful closure of sequenced genome sequences challenging (Frangeul et al., 2008). The biological causes and consequences of this unique genomic architecture, however, remain unexplained.

First reported in maize in 1950 (McClintock, 1950), transposable elements have been identified in genomes across all domains of life and more recently have been implicated in not only driving evolution, but also epigenetics of eukaryotic organisms (Slotkin and Martienssen, 2007). To date, the factors driving transposase expression have not been well defined. The data presented herein suggest that nutrient availability and chemistry may play a role in regulating activity for 11 of the 21 major insertion sequence elements and associated transposase families found in the M. aeruginosa genome (Kaneko et al., 2007). Significantly increased expression occurred most frequently for transposases from the family IS200/IS605, suggesting not only that nutrient limitation may regulate transposase gene expression, but that it specifically targets expression of a single family. This family of transposases is also widespread in the genomes of multiple pathogenic bacteria (Gibert et al., 1990): similar behavior of these elements among pathogens and proteomic identification of transposases in endosymbionts and an acid mine drainage community may have ramifications for the ability of such organisms to infect a host or adapt to an environment (Chao et al., 1983; Ram et al., 2005; Kleiner et al., 2013). Homologs of the IS200/605 family have been identified across both eukaryotes and eukaryote-infecting viruses, suggesting these results may have implications in all domains of life (Bao and Jurka, 2013).

Somewhat surprisingly, prior transcriptome (both microarray and RNA-seq) analyses of Microcystis spp. transcriptomics make no reference to differential expression of transposases across light/dark and nutrient exposure treatments (Straub et al., 2011; Harke and Gobler, 2013). A re-examination of previously published data for Microcystis strain LE-3 grown under N-reduced (nitrate) conditions showed 127 transposase genes differentially expressed, with 122 showing increased expression relative to the control (Harke and Gobler, 2013). Other treatments in that study were unfortunately too dissimilar to compare with our work because of differences in nutrient concentrations and chemistries.

The abundance and expression of transposases within the genome of $M$. aeruginosa NIES 843 could have a substantial impact on genomic architecture of individual cells (Figure 6). Indeed, there are only four expansive regions ( $>150 \mathrm{~kb}$ ) where no transposase genes exist (Figure 6, I-IV and Supplementary Table 3). Within these regions are large numbers of hypothetical proteins, genes involved in cobalamin biosynthesis, nitrogen transport and metabolism (Figure 6 I-III), and DNA and RNA polymerase subunit proteins (Figure 6, II-III). Region II contains all genes encoding for the 5S, 16S and 23S ribosomal subunits and 10 genes involved in phosphate transport (Figure 6). As many of these genes are 


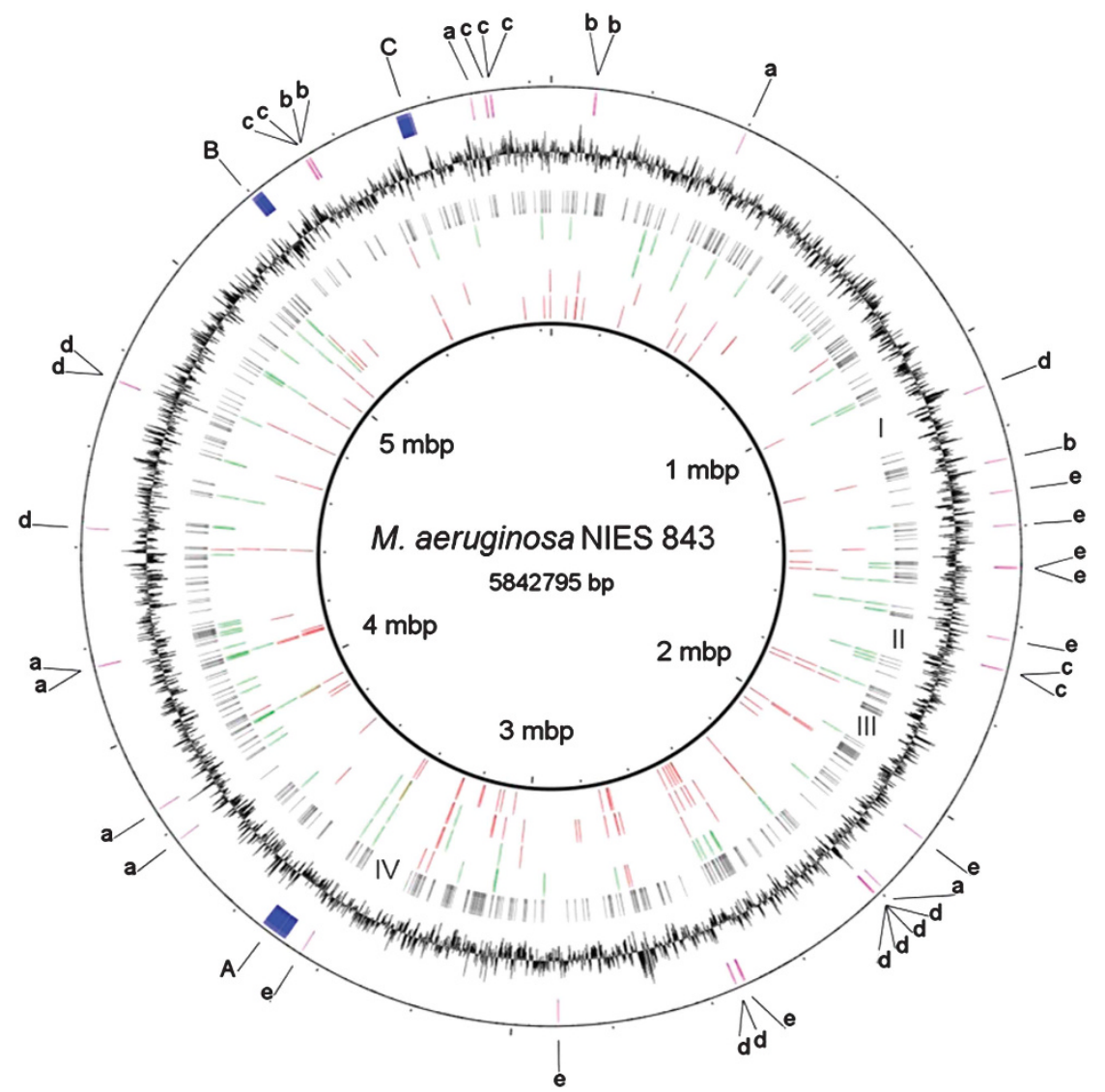

Figure 6 Genome map of M. aeruginosa NIES 843. Inner four rings show locations of significantly increased (green) or decreased (red) expression of transposase genes for the P-reduced (innermost), N-reduced (nitrate), N-reduced (ammonium) and N-reduced (urea) treatments. Location of all transposase genes in the genome in ring five and GC content is located in ring six. The outermost ring shows locations of ure $A-G(\mathrm{a}), \operatorname{hox} F-Y(\mathrm{~b}), \operatorname{hyp} A-F(\mathrm{c}), \operatorname{cpcA} 1-G(\mathrm{~d})$ and putative nif genes (e); mcyA-J (A), aerD-L (B) and mcnA-G (C). Gaps in transposase placement are highlighted as I-IV.

considered essential for viability, it is not surprising that they exist in genome regions free from interference by active insertion sequence elements.

The genome of $M$. aeruginosa is also unique in that many genes, which are canonically organized in other cyanobacteria as single operons (Wang et al., 2012), are scattered throughout as individual open reading frames (Figures 6a-e; Supplementary Table 3). Examples include genes involved in construction of the urease enzyme complex (ureA-G; Figure 6a), both (hoxEFHUY; Figure 6b; hypA-F; Figure 6c) hydrogenases, phycocyanin synthesis genes (cpcA1-G; Figure 6d) and even the genes annotated as being involved in nitrogen fixation (Figure 6e). The mosaic and highly plastic nature of the $M$. aeruginosa NIES 843 genome is suggestive of the ability to produce subpopulations of cells with alternate genomic architectures, and potentially evolutionary trajectories, in response to environmental conditions.

In contrast, genes involved in synthesis of nonribosomal secondary metabolites appear to have maintained the operon architecture observed in other cyanobacterial genomes (Figures 6A-C). Regulation of synthetase genes for microcystins (Figure 6A), aeruginosins (Figure 6B) and cyanopeptolins (Figure 6C) (Kaneko et al., 2007; Ishida et al., 2009) is likely tied to a need for juxtaposition of these gene products to each other for proper synthesis: rearrangements leading to a loss of function likely means the entire cassette would be quickly lost from the genome. In part, this may explain the absence of these capabilities in some isolates: although intact, the genetic cassettes support selection for organisms producing these compounds, allowing the persistence of these capabilities within the genome. Rearrangement of these genes (which need to be proximal for product assembly) removes the positive selection that maintains the rest of the cassette. Indeed, within the presented data, the most highly expressed transposases for the N-reduced treatments were those located proximal to the microcystin cassette that is present in only a subset of natural communities (Rinta-Kanto et al., 2009). Similar activity of transposable elements has been implicated in the loss of microcystin biosynthesis genes in Planktothrix strains (Christiansen et al., 2008).

Ecologically, these drivers of genomic architecture may be exacerbated by changing land use practices: urea has become the dominant nitrogenous fertilizer 
during the past three decades-a period where we have seen expansive proliferations of Microcystis spp. (Glibert et al., 2006). Moreover, the potential role of nutrients as a regulatory mechanism for transposase expression may apply more broadly across microbial life given the wide distribution of the IS200/IS605 family. Indeed, a limited number of other organisms (both bacteria and archaea) have been shown to experience increased transposase expression under various environmental conditions, including $\mathrm{N}$ variability (Hewson et al., 2009; Jäger et al., 2009). This suggests that anthropogenic nutrient loading may not just shape the biomass and evolutionary trajectories of single organisms, but entire microbial communities.

Our observations illustrate a possible evolutionary response that would allow for survival and dominance across the range of freshwater system Microcystis inhabits. Because these data arose from an exploratory transcriptomic study, it highlights the utility of so-called '-omics' tools for hypothesis development for a system of interest. Future testing of these hypotheses is necessary to resolve how biogeochemical conditions can drive evolution (that is, genomic rearrangements) in Microcystis, as increased transcription of transposase genes may not translate to enzymatic activity or genomic rearrangements (Schmitz-Esser et al., 2011). Moreover, these hypotheses require particular testing in field studies with natural populations, as variations in natural communities may shed further light on how biogeochemistry shapes not only community structure but evolutionary trajectory.

\section{Conflict of Interest}

The authors declare no conflict of interest.

\section{Acknowledgements}

We thank Dr GL Boyer, Dr LJ Hauser, Dr NC VerBerkmoes, Dr RL Hettich, Dr GS Bullerjahn and Dr RML McKay for support and meaningful ideas and discussion. We also thank Shafer Belisle, Chad Effler and Justine Schmidt for their assistance. This project was supported by grants from the National Science Foundation (IOS 0841918 and DEB 1240870 to SWW; and OCE 1233964 and OCE 1208784 to SRC) and a UT/ORNL Science Alliance JDRD award to SWW. MMS was supported by a Wallace-Dean fellowship from the University of Tennessee.

\section{References}

Abraham P, Adams R, Giannone RJ, Kalluri U, Ranjan P, Erickson B et al. (2011). Defining the boundaries and characterizing the landscape of functional genome expression in vascular tissues of Populus using shotgun proteomics. J Proteome Res 11: 449-460.
Alikhan N-F, Petty N, Ben Zakour N, Beatson S. (2011). BLAST ring image generator (BRIG): simple prokaryote genome comparisons. BMC Genomics 12: 402.

Baggerly KA, Deng L, Morris JS, Aldaz CM. (2003). Differential expression in SAGE: accounting for normal between-library variation. Bioinformatics 19: 1477-1483.

Bao W, Jurka J. (2013). Homologues of bacterial TnpB_IS605 are widespread in diverse eukaryotic transposable elements. Mobile DNA 4: 12.

Berman T. (1970). Alkaline phosphatases and phosphorus availability in Lake Kinneret. Limnol Oceanogr 15: 663-674.

Berman T, BÃ (C)chemin C, Maestrini SY. (1999). Release of ammonium and urea from dissolved organic nitrogen in aquatic ecosystems. Aquat Microb Ecol 16: 295-302.

Chao L, Vargas C, Spear BB, Cox EC. (1983). Transposable elements as mutator genes in evolution. Nature 303: 633-635.

Christiansen G, Molitor C, Philmus B, Kurmayer R. (2008). Nontoxic strains of cyanobacteria are the result of major gene deletion events induced by a transposable element. Mol Biol Evol 25: 1695-1704.

Clasquin MF, Melamud E, Rabinowitz JD. (2012). LC-MS data processing with MAVEN: a metabolomic analysis and visualization engine. Curr Protoc Bioinformatics 14.11.1-14.11.23.

Davis T, Harke MJ, Marcoval MA, Goleski J, Orano-Dawson C, Berry DL et al. (2010). Effects of nitrogenous compounds and phosphorus on the growth of toxic and non-toxic strains of Microcystis during cyanobacterial blooms. Aquat Microb Ecol 61: 149-162.

DeBruyn JM, Leigh-Bell JA, McKay RML, Bourbonniere RA, Wilhelm SW. (2004). Microbial distributions and the impact of phosphorus on bacterial activity in Lake Erie. J Great Lak Res 30: 166-183.

Dillies M-A, Rau A, Aubert J, Hennequet-Antier C, Jeanmougin M, Servant N et al. (2013). A comprehensive evaluation of normalization methods for Illumina high-throughput RNA sequencing data analysis. Brief Bioinform 14: 671-683.

Dodds WK, Bouska WW, Eitzmann JL, Pilger TJ, Pitts KL, Riley AJ et al. (2008). Eutrophication of U.S. freshwaters: analysis of potential economic damages. Environ Sci Tech 43: 12-19.

Dolman AM, Rücker J, Pick FR, Fastner J, Rohrlack T, Mischke U et al. (2012). Cyanobacteria and cyanotoxins: the influence of nitrogen versus phosphorus. PLoS One 7: e38757.

Donald DB, Bogard MJ, Finlay K, Leavitt PR. (2011). Comparative effects of urea, ammonium, and nitrate on phytoplankton abundance, community composition, and toxicity in hypereutrophic freshwaters. Limnol Oceanogr 56: 2161-2175.

Downing JA, Watson SB, McCauley E. (2001). Predicting cyanobacteria dominance in lakes. Can J Fish Aquat Sci 58: 1905-1908.

Frangeul L, Quillardet P, Castets A-M, Humbert J-F, Matthijs H, Cortez D et al. (2008). Highly plastic genome of Microcystis aeruginosa PCC 7806, a ubiquitous toxic freshwater cyanobacterium. BMC Genomics 9: 274.

Gibert I, Barbé J, Casadesús J. (1990). Distribution of insertion sequence IS200 in Salmonella and Shigella. J Gen Microbiol 136: 2555-2560.

Glibert P, Harrison J, Heil C, Seitzinger S. (2006). Escalating worldwide use of urea - a global change 
contributing to coastal eutrophication. Biogeochemistry 77: 441-463.

Gobler CJ, Berry DL, Dyhrman ST, Wilhelm SW, Salamov A, Lobanov AV et al. (2011). Niche of harmful alga Aureococcus anophagefferens revealed through ecogenomics. Proc Natl Acad Sci USA 108: 4352-4357.

Harke M, Berry D, Ammerman J, Gobler C. (2012). Molecular response of the bloom-forming cyanobacterium, Microcystis aeruginosa, to phosphorus limitation. Microb Ecol 63: 188-198.

Harke M, Gobler C. (2013). Global transcriptional responses of the toxic cyanobacterium, Microcystis aeruginosa, to nitrogen stress, phosphorus stress, and growth on organic matter. PLoS One 8: e69834.

Healey FP, Hendzel LL. (1979). Indicators of phosphorus and nitrogen deficiency in five algae in culture. J Fish Res Board Can 36: 1364-1369.

Hewson I, Poretsky RS, Beinart RA, White AE, Shi T, Bench SR et al. (2009). In situ transcriptomic analysis of the globally important keystone $\mathrm{N}_{2}$-fixing taxon Crocosphaera watsonii. ISME J 3: 618-631.

Hotto AM, Satchwell MF, Berry DL, Gobler CJ, Boyer GL. (2008). Spatial and temporal diversity of microcystins and microcystin-producing genotypes in Oneida Lake, NY. Harmful Algae 7: 671-681.

Humbert J-F, Barbe V, Latifi A, Gugger M, Calteau A, Coursin T et al. (2013). A tribute to disorder in the genome of the bloom-forming freshwater cyanobacterium Microcystis aeruginosa. PLoS One 8: e70747.

Ichimura T. (1971). Sexual cell division and conjugationpapilla formation in sexual reproduction of Closterium stringosum. Proceedings of the 7 th International Seaweed Symposium, 1971, Sappora, Japan.

Ishida $K$, Welker $M$, Christiansen $G$, Cadel-Six S, Bouchier C, Dittmann E et al. (2009). Plasticity and evolution of aeruginosin biosynthesis in cyanobacteria. Appl Environ Microbiol 75: 2017-2026.

Ivanikova NV, McKay RML, Bullerjahn GS, Sterner RW. (2007). Nitrate utilization by phytoplankton in Lake Superior is impaired by nutrient (P, Fe) availability and season light limitation - a cyanobacterial bioreporter study. J Phycol 43: 475-484.

Jäger D, Sharma CM, Thomsen J, Ehlers C, Vogel J, Schmitz RA. (2009). Deep sequencing analysis of the Methanosarcina mazei Gö1 transcriptome in response to nitrogen availability. Proc Natl Acad Sci USA 106: 21878-21882.

Kaneko T, Nakajima N, Okamoto S, Suzuki I, Tanabe Y, Tamaoki $\mathrm{M}$ et al. (2007). Complete genomic structure of the bloom-forming toxic cyanobacterium Microcystis aeruginosa NIES-843. DNA Res 14: 247-256.

Kleiner M, Young JC, Shah M, VerBerkmoes NC, Dubilier N. (2013). Metaproteomics reveals abundant transposase expression in mutualistic endosymbionts. mBio 4: e00223-13.

Lin S, Haas S, Zemojtel T, Xiao P, Vingron M, Li R. (2011). Genome-wide comparison of cyanobacterial transposable elements, potential genetic diversity indicators. Gene 473: 139-149.

Lu WY, Clasquin MF, Melamud E, Amador-Noguez D, Caudy AA, Rabinowitz JD. (2010). Metabolomic analysis via reversed-phase ion-pairing liquid chromatography coupled to a stand alone Orbitrap mass spectrometer. Anal Chem 82: 3212-3221.

Mackerras AH, Smith GD. (1986). Urease activity of the cyanobacterium Anabaena cylindrica. J Gen Microbiol 132: $2749-2752$.
McClintock B. (1950). The origin and behavior of mutable loci in maize. Proc Natl Acad Sci USA 36: 344-355.

Melamud E, Vastag L, Rabinowitz JD. (2010). Metabolomic analysis and visualization engine for LC-MS data. Anal Chem 82: 9818-9826.

Mortazavi A, Williams BA, McCue K, Schaeffer L, Wold B. (2008). Mapping and quantifying mammalian transcriptomes by RNA-Seq. Nat Meth 5: 621-628.

Myklestad S, Sakshaug E. (1983). Alkaline phosphatase activity of Skeletonema costatum populations in the Trondheimsfjord. J Plank Res 5: 557-564.

Nausch M. (1998). Alkaline phosphatase activities and the relationship to inorganic phosphate in the Pomeranian Bight (southern Baltic Sea). Aquat Microb Ecol 16: 87-94.

Neddermann K, Nausch M. (2004). Effects of organic and inorganic nitrogen compounds on the activity of bacterial alkaline phosphatase. Aquat Ecol 38: 475-484.

Paerl HW, Xu H, McCarthy MJ, Zhu G, Qin B, Li Y et al. (2011). Controlling harmful cyanobacterial blooms in a hyper-eutrophic lake (Lake Taihu, China): the need for a dual nutrient (N \& P) management strategy. Water Res 45: 1973-1983.

Paterson M, Schindler D, Hecky R, Findlay D. (2011). Comment: Lake 227 shows clearly that controlling inputs of nitrogen will not reduce or prevent eutrophication of lakes. Limnol Oceanogr 56: 1545-1547.

Purvina S, Bechemin C, Balode M, Verite C, Arnaud C, Maerstrini SY. (2010). Release of available nitrogen from river-discharged dissolved organic matter by heterotrophic bacteria associated with the cyanobacterium Microcystis aeruginosa. Estonian J Ecol 59: 184-196.

Ram RJ, VerBerkmoes NC, Thelen MP, Tyson GW, Baker BJ, Blake RC et al. (2005). Community proteomics of a natural microbial biofilm. Science 308: 1915-1920.

Rinta-Kanto JM, Konopko EA, DeBruyn JM, Bourbonniere RA, Boyer GL, Wilhelm SW. (2009). Lake Erie Microcystis: relationship between microcystin production, dynamics of genotypes and environmental parameters in a large lake. Harmful Algae 8: 665-673.

Schindler DW. (1977). Evolution of phosphorus limitation in lakes. Science 195: 260-262.

Schmitz-Esser S, Penz T, Spang A, Horn M. (2011). A bacterial genome in transition - an exceptional enrichment of IS elements but lack of evidence for recent transposition in the symbiont Amoebophilus asiaticus. BMC Evol Biol 11: 270.

Scott JT, McCarthy MJ. (2010). Nitrogen fixation may not balance the nitrogen pool in lakes over timescales relevant to eutrophication management. Limnol Oceanogr 55: 1265-1270.

Segata N, Waldron L, Ballarini A, Narasimhan V, Jousson O, Huttenhower C. (2012). Metagenomic microbial community profiling using unique cladespecific marker genes. Nat Meth 9: 811-814.

Shen H, Song L. (2007). Comparative studies on physiological responses to phosphorus in two phenotypes of bloom-forming Microcystis. Hydrobiologia 592: 475-486.

Slotkin RK, Martienssen R. (2007). Transposable elements and the epigenetic regulation of the genome. Nat Rev Genet 8: 272-285. 
Spura J, Christian Reimer L, Wieloch P, Schreiber K, Buchinger S, Schomburg D. (2009). A method for enzyme quenching in microbial metabolome analysis successfully applied to gram-positive and gram-negative bacteria and yeast. Anal Biochem 394: 192-201.

Steglich C, Lindell D, Futschik M, Rector T, Steen R, Chisholm S. (2010). Short RNA half-lives in the slow-growing marine cyanobacterium Prochlorococcus. Genome Biol 11: R54.

Straub C, Quillardet P, Vergalli J, de Marsac NT, Humbert J-F. (2011). A day in the life of Microcystis aeruginosa strain PCC 7806 as revealed by a transcriptomic analysis. PLoS One 6: e16208.

Vézie C, Rapala J, Vaitomaa J, Seitsonen J, Sivonen K. (2002). Effect of nitrogen and phosphorus on growth of toxic and nontoxic Microcystis strains and on intracellular microcystin concentrations. Microb Ecol 43: 443-454.

Wang H, Sivonen K, Rouhiainen L, Fewer D, Lyra C, Rantala-Ylinen A et al. (2012). Genome-derived insights into the biology of the hepatotoxic bloomforming cyanobacterium Anabaena sp. strain 90. BMC Genomics 13: 613.

Welschmeyer NA. (1994). Fluorometric analysis of chlorophyll-a in the presence of chlorophyll-B and pheopigments. Limnol Oceanogr 39: 1985-1992.

Wilhelm SW, DeBruyn JM, Gillor O, Twiss MR, Livingston K, Bourbonniere RA et al. (2003). Effect of phosphorus amendments on present day plankton communities in pelagic Lake Erie. Aquat Microb Ecol 32: 275-285.

Wurch LL, Haley ST, Orchard ED, Gobler CJ, Dyhrman ST. (2011). Nutrient-regulated transcriptional responses in the brown tide-forming alga Aureococcus anophagefferens. Environ Microbiol 13: 468-481.

Zybailov B, Mosley AL, Sardiu ME, Coleman MK, Florens L, Washburn MP. (2006). Statistical analysis of membrane proteome expression changes in Saccharomyces cerevisiae. J Proteome Res 5: 2339-2347.

Supplementary Information accompanies this paper on The ISME Journal website (http://www.nature.com/ismej) 